\title{
Left ventricular diastolic function and exercise capacity in diabetes
}

\author{
P. Brassard • P. Poirier
}

Received: 19 November 2008 / Accepted: 23 January 2009/Published online: 24 February 2009

(C) Springer-Verlag 2009

\begin{abstract}
Abbreviations
LV Left ventricular

LVDD Left ventricular diastolic dysfunction
\end{abstract}

To the Editor: Gusso et al. [1] have reported interesting findings regarding the impairment of stroke volume responses to submaximal exercise in female adolescent individuals with type 1 and type 2 diabetes compared with non-diabetic controls. The diabetic patients also had reduced maximal oxygen uptake. Although the pathophysiological mechanism(s) underlying the reduction in stroke volume during exercise in these young people with diabetes was outside the scope of the study, the authors suggested that increased myocardial stiffness and/or altered filling characteristics related to diabetes could have been associated with the abnormal stroke volume response and might have limited exercise performance. This is an interesting viewpoint, since whether abnormal exercise capacity is affected by well-controlled, uncomplicated diabetes per se is still a matter of debate.

Diabetes has a negative impact on left ventricular (LV) diastolic function in adults [2]. Interestingly, adult patients with well-controlled, uncomplicated type 2 diabetes and left ventricular diastolic dysfunction (LVDD) have been reported

P. Brassard $(\bowtie)$

Department of Anaesthesia 2041,

Rigshospitalet,

Blegdamsvej 9,

2100 Copenhagen Ø, Denmark

e-mail: patrice.brassard@gmail.com

P. Poirier

Centre de recherche de l'Hôpital Laval,

Institut universitaire de cardiologie et de pneumologie,

Université Laval,

Quebec, Canada to have a reduced exercise performance compared with controls [3]. Aerobic exercise training normalises LVDD and enhances maximal oxygen uptake in well-controlled, uncomplicated type 2 diabetes [4], suggesting that LVDD could negatively affect exercise performance in these patients. What happens to variables such as stroke volume and cardiac output during exercise in patients with diabetes and an abnormal LV diastolic function or LVDD is less clear. Gusso et al. [1] provide some potential pathophysiological insights that bring our understanding one step further with their results obtained during submaximal exercise. They suggest that early cardiac impairments (such as LV diastolic function-related abnormalities) could partly explain the blunted stroke volume responses to submaximal exercise while reducing exercise capacity in young female patients with diabetes. This is an appealing explanation.

However, abnormalities in LV diastolic function or LVDD are also present in young and adult individuals with uncomplicated obesity [5, 6]. Even if diabetes has an independent effect on LV diastolic function in adults, it would have been interesting if Gusso et al. [1] had provided echocardiographic data as well as data on the prevalence of LVDD in each group. This would enable identification of any difference in the severity of abnormalities in LV diastolic function and in the prevalence of LVDD between groups, which could also have an impact on stroke volume. In addition, although it is difficult to evaluate the duration of diabetes precisely, especially in type 2 diabetes, disease duration could have influenced the severity of abnormalities in LV diastolic function in their diabetic groups.

As highlighted by Gusso et al., the reduction in exercise capacity in their individuals with diabetes could have also been related to impairment in autonomic response to exercise limiting maximal heart rate. Since LVDD has been linked to abnormal heart rate variability in patients with type 2 diabetes 
[7], LVDD could eventually affect exercise capacity indirectly via the associated impairment in cardiac autonomic function.

Their study highlights additional issues. Since it has already been shown that sex influences LV diastolic function in young people [8], are the results applicable to young male patients with diabetes? In addition, knowing that patients with type 1 diabetes were not obese, what would be the relative contribution of obesity in the presence of abnormalities in LV diastolic function in these young people with type 1 diabetes vs those with type 2 diabetes? Several issues need to be clarified regarding the involvement of an abnormal LV diastolic function in the reduced exercise capacity in young and adult patients with diabetes. The article by Gusso et al. [1] adds important details to this fascinating story.

\section{References}

1. Gusso S, Hofman P, Lalande S, Cutfield W, Robinson E, Baldi JC (2008) Impaired stroke volume and aerobic capacity in female adolescents with type 1 and type 2 diabetes mellitus. Diabetologia 51:1317-1320
2. Baldi JC, Aoina JL, Whalley GA et al (2006) The effect of type 2 diabetes on diastolic function. Med Sci Sports Exerc 38:13841388

3. Poirier P, Garneau C, Bogaty P et al (2000) Impact of left ventricular diastolic dysfunction on maximal treadmill performance in normotensive subjects with well-controlled type 2 diabetes mellitus. Am J Cardiol 85:473-477

4. Brassard P, Legault S, Garneau C, Bogaty P, Dumesnil JG, Poirier $P$ (2007) Normalization of diastolic dysfunction in type 2 diabetics after exercise training. Med Sci Sports Exerc 39:1896-1901

5. Poirier P, Giles TD, Bray GA et al (2006) Obesity and cardiovascular disease: pathophysiology, evaluation, and effect of weight loss: an update of the 1997 American Heart Association Scientific Statement on Obesity and Heart Disease from the Obesity Committee of the Council on Nutrition, Physical Activity, and Metabolism. Circulation 113:898-918

6. Sharpe JA, Naylor LH, Jones TW et al (2006) Impact of obesity on diastolic function in subjects $\leq 16$ years of age. Am J Cardiol 98:691-693

7. Poirier P, Bogaty P, Philippon F, Garneau C, Fortin C, Dumesnil JG (2003) Preclinical diabetic cardiomyopathy: relation of left ventricular diastolic dysfunction to cardiac autonomic neuropathy in men with uncomplicated well-controlled type 2 diabetes. Metabolism 52:1056-1061

8. Kapuku GK, Davis HC, Shah N, McMillan AM, Harshfield GA (2008) Gender differences in diastolic function among youth. Pediatr Cardiol 29:102-107 